\title{
Hölder quasicontinuity of Sobolev functions on metric spaces
}

\author{
Piotr Hajłasz and Juha Kinnunen
}

\begin{abstract}
We prove that every Sobolev function defined on a metric space coincides with a Hölder continuous function outside a set of small Hausdorff content or capacity. Moreover, the Hölder continuous function can be chosen so that it approximates the given function in the Sobolev norm. This is a generalization of a result of Malý [Ma1] to the Sobolev spaces on metric spaces [H1].
\end{abstract}

\section{Introduction.}

The classical Luzin theorem asserts that every measurable function is continuous if it is restricted to the complement of a set of arbitrary small measure. If the function is more regular, then it is natural to expect that Luzin's theorem can be refined. One important class of functions are the Sobolev functions. It is known that every Sobolev function, after a redefinition on a set of measure zero, is continuous when restricted to the complement of a set of arbitrary small capacity. This is a capacitary version of Luzin's theorem. On the other hand, if we restrict the function to the complement of a slightly larger set, we obtain more regularity, see $[\mathrm{BH}],[\mathrm{CZ}],[\mathrm{Li}],[\mathrm{MZ}]$ and $[\mathrm{Z}]$. We are interested in the Hölder continuity of the restriction. Indeed, a Sobolev function coincides with a Hölder continuous function on the complement of a set of arbitrary small capacity. Moreover, the Hölder continuous function can be chosen so that it belongs to the Sobolev space and it approxi- 
mates the given function in the Sobolev norm. This phenomenon was first observed by Malý [Ma1] in the Euclidean case. Malý's result plays a crucial role in the refined versions of the change of variable formula for the Sobolev functions, see [Ma2] and [MM].

It is possible to define the first order Sobolev space and to develop a capacity theory on an arbitrary metric space which is equipped with a doubling measure, see $[\mathrm{H} 1]$ and $[\mathrm{KM}]$. Hence all notions in refined Luzin's and Malý's theorems make sense also in the metric context. Indeed, the capacitary version of Luzin's theorem holds, see [KM]. The purpose of this paper is to generalize Malý's result to metric spaces. As a by-product we obtain a new proof for the Euclidean case. Malý's argument is based on the representation of the Sobolev functions by the Bessel potentials and it does not generalize to the metric setting. Our approach is based on pointwise estimates for the Sobolev functions. In fact, we obtain slightly better estimates for the exponent of the Hölder continuity and the size of the exceptional set than Malý.

The fundamental fact in our proof is that the oscillation of a Sobolev function is controlled pointwise by the fractional maximal function of the derivative, see [H2], [HM, Lemma 4]. If the fractional maximal function is bounded, then the function is Hölder continuous. This is Morrey's lemma. A generalization of Morrey's lemma to metric spaces has been studied in [MS1]. Their main result follows from our pointwise estimates.

If the fractional maximal function is not bounded, the function is Hölder continuous when restricted to the set where the maximal function is small. The classical weak type inequalities give estimates for the Hausdorff content and for the capacity of the exceptional set. As a consequence, we obtain that, after a redefinition on a set of measure zero, a Sobolev function coincides with a Hölder continuous function outside a very small set. The obtained function can be easily extended to the Hölder continuous function on the whole space. The main problem is to construct the extension so that it belongs to the Sobolev space and approximates the given function in the Sobolev norm. Instead of using the McShane extension [Mc] we use a Whitney type extension. However, in our case it should be rather called Whitney smoothing, since instead of extending the function we smooth it in the bad set. The method of our paper can be generalized to higher order derivatives in the Euclidean case, see [BHS].

One of the most important applications of Sobolev spaces on metric spaces are the Sobolev spaces associated to the vector fields. If $V=$ 
$\left\{X_{1}, X_{2}, \ldots, X_{k}\right\}$ is a family of vector fields satisfying Hörmander's condition on $\mathbb{R}^{n}$, and $W_{V}^{1, p}$ is the closure of $C^{\infty}\left(\mathbb{R}^{n}\right)$ in the norm

$$
\|f\|_{W_{V}^{1, p}}=\left(\|f\|_{L^{p}}^{p}+\|X f\|_{L^{p}}^{p}\right)^{1 / p}
$$

where

$$
|X f|=\left(\sum_{i=1}^{k}\left|X_{i} f\right|^{2}\right)^{1 / 2}
$$

then $W_{V}^{1, p}$ is equivalent to the Sobolev space on metric space $\mathbb{R}^{n}$ with the Carnot-Carathéodory metric and the Lebesgue measure. This can be deduced from the Poincaré inequality of Jerison, see [CDG], $[\mathrm{J}]$, [FLW2], the fact that the Lipschitz functions with respect to the Carnot-Carathéodory metric belong to $W_{V}^{1, p}$, see [FSS], [GN2], and an approximation argument similar to that we use in the proof of our main result. For related results, see [FHK], [FLW1], [GN1], [HK2] and [Vo]. In this paper we work in general metric spaces and no knowledge in the theory of vector fields satisfying Hörmander's condition is required. For other papers related to the Sobolev spaces on metric spaces, see [HeK], $[\mathrm{HM}],[\mathrm{K}],[\mathrm{KM}],[\mathrm{KMc}]$ and $[\mathrm{Se}]$.

The outline of our paper is the following. Section 2 contains some results on the maximal functions and measure theory. In Section 3 we recall the definitions of the Sobolev spaces and the capacity on metric spaces. We also give two characterizations of Sobolev spaces. The first characterization is in terms of Poincaré inequalities and the second is a generalization of a result of Calderón [C]. Section 4 is devoted to study the set of Lebesgue's points of a Sobolev function. The main result (Theorem 5.3) is presented in Section 5.

Our notation is fairly standard. By $B(x, r)$ we denote an open ball with the center $x$ and the radius $r$. The symbol $\chi_{E}$ stands for the characteristic function of the set $E$. The average value of $f$ over the ball $B(x, r)$ is denoted by

$$
f_{B(x, r)}=f_{B(x, r)} f d \mu=\frac{1}{\mu(B(x, r))} \int_{B(x, r)} f d \mu .
$$

Various positive constants are denoted by $c$; they may change even on the same line. The dependence on the parameters is expressed, for example, by $c=c(n, p)$. We say that two quantities are comparable, and denote $A \approx B$, if there is a constant $c \geq 1$ such that $A / c \leq B \leq c A$. 


\section{Measure theory.}

In this section we collect some basic results concerning measure theory and the maximal functions. With minor changes all the results of this section are standard, see [Ch], [CW], [St].

Throughout the paper $(X, d)$ is a metric space and $\mu$ a non-negative Borel regular outer measure on $X$ which is finite on bounded sets. We also assume that $\mu$ is doubling in the sense that

$$
\mu(B(x, 2 r)) \leq C_{d} \mu(B(x, r)),
$$

whenever $x \in X$ and $r>0$. The constant $C_{d}$ is called the doubling constant.

The first result states that the doubling condition gives a lower bound for the growth of the measure of a ball.

Proposition 2.1. Suppose that $\mu$ is a doubling measure on $(X, d)$. If $Y \subset X$ is a bounded set, then

$$
\mu(B(x, r)) \geq(2 \operatorname{diam} Y)^{-n} \mu(Y) r^{n},
$$

for $n=\log _{2} C_{d}, x \in Y$ and $0<r \leq \operatorname{diam} Y$. Here $C_{d}$ is the doubling constant of $\mu$.

In this paper we keep the triple $(X, d, \mu)$ fixed and $n$ always refers to the exponent in (2.2).

Let $0 \leq \alpha<\infty$ and $R>0$. The fractional maximal function of a locally integrable function $f$ is defined by

$$
\mathcal{M}_{\alpha, R} f(x)=\sup _{0<r<R} r^{\alpha} f_{B(x, r)}|f| d \mu,
$$

If $Y \subset X$, then we denote $\mathcal{M}_{\alpha, Y}=\mathcal{M}_{\alpha, \operatorname{diam} Y}$. For $R=\infty$, we write $\mathcal{M}_{\alpha, \infty}=\mathcal{M}_{\alpha}$. If $\alpha=0$, we obtain the Hardy-Littlewood maximal function and we write $\mathcal{M}_{0}=\mathcal{M}$. By the Hardy-Littlewood maximal theorem $\mathcal{M}$ is bounded in $L^{p}$ provided $1<p \leq \infty$. For $p=1$ we have a weak type inequality.

Proposition 2.3. Under the above assumptions

$$
\mu(\{x \in X: \mathcal{M} f(x)>\lambda\}) \leq \frac{c}{\lambda}\|f\|_{L^{1}(X)}, \quad \lambda>0,
$$


and

$$
\|\mathcal{M} f\|_{L^{p}(X)} \leq c\|f\|_{L^{p}(X)}, \quad 1<p \leq \infty
$$

It is easy to verify that the set

$$
E_{\lambda}=\left\{x \in X: \mathcal{M}_{\alpha} f(x)>\lambda\right\}, \quad \lambda>0,
$$

is open. Next we would like to get some estimates for the size of the set $E_{\lambda}$. To this end, recall that the Hausdorff $s$-content of $E \subset X$ is defined by

$$
\mathcal{H}_{\infty}^{s}(E)=\inf \left\{\sum_{i=1}^{\infty} r_{i}^{s}: E \subset \bigcup_{i=1}^{\infty} B\left(x_{i}, r_{i}\right)\right\}
$$

It is easy to see that $\mathcal{H}_{\infty}^{s}(E)=0$ if and only if $\mathcal{H}^{s}(E)=0$, where $\mathcal{H}^{s}$ denotes the Hausdorff $s$-measure.

By the standard Vitali covering argument [CW, p. 69] we obtain the following weak type inequality for the fractional maximal function, see [BZ, Lemma 3.2], [St, Theorem 3.3].

Lemma 2.6. Suppose that $f \in L^{1}(X)$ and let $Y \subset X$ be a bounded set with $\mu(Y)>0$. Let $n$ be as in (2.2) and $0 \leq \alpha<n$. Then

$$
\mathcal{H}_{\infty}^{n-\alpha}\left(\left\{x \in Y: \mathcal{M}_{\alpha, Y} f(x)>\lambda\right\}\right) \leq \frac{c}{\lambda} \int_{X}|f| d \mu, \quad \lambda>0
$$

with $c=5^{n-\alpha}(2 \operatorname{diam} Y)^{n} \mu(Y)^{-1}$.

\section{Sobolev space and capacity.}

Let $u: X \longrightarrow[-\infty, \infty]$ be $\mu$-measurable. We denote by $D(u)$ the set of all $\mu$-measurable functions $g: X \longrightarrow[0, \infty]$ such that

$$
|u(x)-u(y)| \leq d(x, y)(g(x)+g(y)),
$$

almost everywhere. By saying that inequality (3.1) holds almost everywhere we mean that there exists $N \subset X$ with $\mu(N)=0$ such that (3.1) holds for every $x, y \in X \backslash N$. 
A function $u \in L^{p}(X)$ belongs to the Sobolev space $W^{1, p}(X)$, $1<p \leq \infty$, if $D(u) \cap L^{p}(X) \neq \varnothing$. The space $W^{1, p}(X)$ is endowed with the norm

$$
\|u\|_{W^{1, p}(X)}=\left(\|u\|_{L^{p}(X)}^{p}+\|u\|_{L^{1, p}(X)}^{p}\right)^{1 / p}
$$

where

$$
\|u\|_{L^{1, p}(X)}=\inf _{g \in D(u)}\|g\|_{L^{p}(X)}
$$

With this norm $W^{1, p}(X)$ is a Banach space.

If $X=\Omega \subset \mathbb{R}^{n}$ is an open bounded domain with a Lipschitz boundary, $d$ is the Euclidean metric and $\mu$ is the Lebesgue measure, then the above definition is equivalent to the standard definition of the Sobolev space $W^{1, p}(\Omega)$. Moreover, $\|u\|_{L^{1, p}} \approx\|\nabla u\|_{L^{p}}$, see [H1]. This explains our notation: $D(u)$ corresponds to the set of the "generalized" gradients of $u$.

The above definition of the Sobolev space on a metric space is due to the first author [H1]. If $p=1$, then the above metric definition in no longer equivalent to the standard definition, see [H2]. For that reason we exclude $p=1$.

We present two characterizations of the Sobolev space on a metric space, see [FHK], [FLW1], [HK2], [KMc] for related results. To this end, we need yet another maximal function.

Let $0<\beta<\infty$ and $R>0$. The fractional sharp maximal function of a locally integrable function $f$ is defined by

$$
f_{\beta, R}^{\#}(x)=\sup _{0<r<R} r^{-\beta} f_{B(x, r)}\left|f-f_{B(x, r)}\right| d \mu,
$$

If $R=\infty$ we simply write $f_{\beta}^{\#}(x)$.

Theorem 3.4. Let $1<p \leq \infty$. Then the following three conditions are equivalent.

1) $u \in W^{1, p}(X)$.

2) $u \in L^{p}(X)$ and there is $g \in L^{p}(X), g \geq 0$, such that the Poincaré inequality

$$
f_{B(x, r)}\left|u-u_{B(x, r)}\right| d \mu \leq c r f_{B(x, r)} g d \mu
$$


holds for every $x \in X$ and $r>0$.

3) $u \in L^{p}(X)$ and $u_{1}^{\#} \in L^{p}(X)$.

Moreover, we obtain

$$
\|u\|_{L^{1, p}(X)} \approx \inf \left\{\|g\|_{L^{p}(X)}: g \text { satisfies }(3.5)\right\} \approx\left\|u_{1}^{\#}\right\|_{L^{p}(X)}
$$

REMARK. The equivalence of 1) and 2) has been proved in [FLW1] and in the classical Euclidean case the equivalence of 1) and 3) can be found in $[\mathrm{C}]$.

Proof. The implication 1) implies 2) follows by integrating (3.1) twice over the ball.

We prove 2) implies 3). The Poincaré inequality (3.5) implies that

$$
r^{-1} f_{B(x, r)}\left|u-u_{B(x, r)}\right| d \mu \leq c f_{B(x, r)} g d \mu
$$

Hence

$$
u_{1}^{\#}(x) \leq c \mathcal{M} g(x)
$$

for every $x \in X$ and the claim follows from the Hardy-Littlewood maximal theorem (Proposition 2.3).

3 ) implies 1). We need the following lemma which, in the Euclidean case with the Lebesgue measure, has been proved in [DS, Theorem 2.7].

Lemma 3.6. Suppose that $f: X \longrightarrow[-\infty, \infty]$ is locally integrable and let $0<\beta<\infty$. Then there is a constant $c=c\left(\beta, C_{d}\right)$ such that

$$
|f(x)-f(y)| \leq c d(x, y)^{\beta}\left(f_{\beta, 4 d(x, y)}^{\#}(x)+f_{\beta, 4 d(x, y)}^{\#}(y)\right),
$$

for almost every $x, y \in X$.

Proof. Let $N$ be the the complement of the set of Lebesgue points of $f$ in $X$. Fix $x \in X \backslash N, 0<r<\infty$ and denote $B_{i}=B\left(x, 2^{-i} r\right)$, 
$i=0,1, \ldots$ By Lebesgue's theorem $\mu(N)=0$, see $[\mathrm{CW}]$. Then

$$
\begin{aligned}
\left|f(x)-f_{B(x, r)}\right| & \leq \sum_{i=0}^{\infty}\left|f_{B_{i+1}}-f_{B_{i}}\right| \\
& \leq \sum_{i=0}^{\infty} \frac{\mu\left(B_{i}\right)}{\mu\left(B_{i+1}\right)} f_{B_{i}}\left|f-f_{B_{i}}\right| d \mu \\
& \leq c \sum_{i=0}^{\infty}\left(2^{-i} r\right)^{\beta}\left(2^{-i} r\right)^{-\beta} f_{B_{i}}\left|f-f_{B_{i}}\right| d \mu \\
& \leq c r^{\beta} f_{\beta, r}^{\#}(x) .
\end{aligned}
$$

Let $y \in B(x, r) \backslash N$. Then $B(x, r) \subset B(y, 2 r)$ and we obtain

$$
\begin{aligned}
\left|f(y)-f_{B(x, r)}\right| & \leq\left|f(y)-f_{B(y, 2 r)}\right|+\left|f_{B(y, 2 r)}-f_{B(x, r)}\right| \\
& \leq c r^{\beta} f_{\beta, 2 r}^{\#}(y)+f_{B(x, r)}\left|f-f_{B(y, 2 r)}\right| d \mu \\
& \leq c r^{\beta} f_{\beta, 2 r}^{\#}(y)+c f_{B(y, 2 r)}\left|f-f_{B(y, 2 r)}\right| d \mu \\
& \leq c r^{\beta} f_{\beta, 2 r}^{\#}(y) .
\end{aligned}
$$

Let $x, y \in X \backslash N, x \neq y$ and $r=2 d(x, y)$. Then $x, y \in B(x, r)$ and hence (3.8) and (3.9) imply that

$$
\begin{aligned}
|f(x)-f(y)| & \leq\left|f(x)-f_{B(x, r)}\right|+\left|f(y)-f_{B(x, r)}\right| \\
& \leq c d(x, y)^{\beta}\left(f_{\beta, 4 d(x, y)}^{\#}(x)+f_{\beta, 4 d(x, y)}^{\#}(y)\right) .
\end{aligned}
$$

This completes the proof.

Now the last implication in Theorem 3.4 follows immediately from Lemma 3.6 and the definition of the Sobolev space. Moreover, the equivalence of the norms follows from the proof. This completes the proof of Theorem 3.4.

Now we state some useful inequalities for the future reference.

Corollary 3.10. Let $u \in W^{1, p}(X), g \in D(u) \cap L^{p}(X)$ and $0 \leq \alpha<1$. Then

$$
u_{1-\alpha, R}^{\#}(x) \leq c \mathcal{M}_{\alpha, R} g(x) \text {, }
$$


for every $R>0$ and $x \in X$. Moreover, we have

$$
|u(x)-u(y)| \leq c d(x, y)^{1-\alpha}\left(\mathcal{M}_{\alpha, 4 d(x, y)} g(x)+\mathcal{M}_{\alpha, 4 d(x, y)} g(y)\right),
$$

for almost every $x, y \in X$.

Proof. The first assertion follows from the Poincaré inequality, since

$$
r^{\alpha-1} f_{B(x, r)}\left|u-u_{B(x, r)}\right| d \mu \leq c r^{\alpha} f_{B(x, r)} g d \mu,
$$

for every $x \in X$ and $0<r<R$. Inequality (3.11) and Lemma 3.6 imply (3.12).

There is a natural capacity in the Sobolev space $W^{1, p}(X)$. The norm (3.2) enables us to define the Sobolev p-capacity of an arbitrary set $E \subset X$ by

$$
\mathrm{C}_{p}(E)=\inf _{u \in \mathcal{A}(E)}\|u\|_{W^{1, p}(X)}^{p},
$$

where the infimum is taken over all admissible functions

$$
\mathcal{A}(E)=\left\{u \in W^{1, p}(X): u \geq 1 \text { on an open neighbourhood of } E\right\} .
$$

This capacity is a monotone and a countably subadditive set function. The rudiments of the capacity theory on metric spaces were developed by the second author with O. Martio in [KM]. By [KM, Theorem 4.6] there is a constant $c=c\left(p, C_{d}\right)$ such that

$$
\mathrm{C}_{p}(B(x, r)) \leq c r^{-p} \mu(B(x, r)), \quad 0<r \leq 1 .
$$

Using the same standard covering argument as in the proof of Lemma 2.6 together with (3.14) and the assumption that the measure is doubling we obtain the following capacitary version of Lemma 2.6.

Lemma 3.15. Suppose that $f \in L^{1}(X)$ and let $1<\alpha<\infty$. Then there is $c=c\left(C_{d}, \alpha\right)$ such that

$$
\mathrm{C}_{\alpha}\left(\left\{x \in X: \mathcal{M}_{\alpha, 1} f(x)>\lambda\right\}\right) \leq \frac{c}{\lambda}\|f\|_{L^{1}(X)}, \quad \lambda>0 .
$$




\section{Lebesgue points.}

A Sobolev function $u \in W^{1, p}(X)$ is defined only up to a set of measure zero, but we define $u$ everywhere in $X$ by

$$
\widetilde{u}(x)=\limsup _{r \rightarrow 0} f_{B(x, r)} u d \mu .
$$

By Lebesgue's theorem not only the limit superior but the limit exists and equals to $u$ almost everywhere. Hence $\widetilde{u}$ coincides with $u$ almost everywhere and gives the same element in $W^{1, p}(X)$. We identify $u$ with $\widetilde{u}$ and omit the tilde in notation.

We recall that $x \in X$ is Lebesgue's point for $u$ if

$$
f_{B(x, r)}|u(y)-u(x)| d \mu(y) \longrightarrow 0
$$

as $r \longrightarrow 0$. Lebesgue's theorem states that almost all points of a $L_{\mathrm{loc}}^{1}(X)$ function are Lebesgue points, see [CW]. If a function belongs to the classical Sobolev space, then we can improve the result and prove that the complement of the set of the Lebesgue points has has small Hausdorff dimension, see [FZ], [Z], [EG]. We generalize this result to the Sobolev spaces on metric spaces.

By (3.8) we have for $\beta>0$

$$
\begin{aligned}
f_{B(x, r)} \mid u(y) & -u(x) \mid d \mu(y) \\
& \leq f_{B(x, r)}\left|u(y)-u_{B(x, r)}\right| d \mu(y)+\left|u_{B(x, r)}-u(x)\right| \\
& \leq c r^{\beta} u_{\beta, r}^{\#}(x) .
\end{aligned}
$$

Letting $r \rightarrow 0$ we see that $x$ is Lebesgue's point for $u$ provided $u_{\beta, 1}^{\#}(x)<$ $\infty$.

We want to estimate the size of the set of the Lebesgue points of $u \in W^{1, p}(X)$. We begin with studying the case $p>n$, where $n$ is the exponent in $(2.2)$.

Proposition 4.3. Suppose that $u \in W^{1, p}(X)$ with $n<p<\infty$. Then $u$ is Hölder continuous on every bounded set in $X$. In particular, every point is a Lebesgue point of $u$. 
Proof. Let $g \in D(u) \cap L^{p}(X)$. It follows from the Hölder inequality and (2.2) that $\mathcal{M}_{n / p} g$ is bounded in every ball. Hence (3.12) implies that $u$ is Hölder continuous with the exponent $1-n / p$ in every ball.

Then we consider the more interesting case $1<p \leq n$.

Theorem 4.4. Suppose that $u \in W^{1, p}(X), g \in D(u) \cap L^{p}(X)$ and $1<p \leq n$. Let $0 \leq \alpha<1$. Then every point for which $\mathcal{M}_{\alpha, 1} g(x)<\infty$ is Lebesgue's point for u. Moreover, the Hausdorff dimension of the complement of the set of the Lebesgue points of $u$ is less than or equal to $n-p$.

Proof. Inequalities (4.2) and (3.11) imply that

$$
f_{B(x, r)}|u(y)-u(x)| d \mu(y) \leq c r^{1-\alpha} u_{1-\alpha, 1}^{\#}(x) \leq c r^{1-\alpha} \mathcal{M}_{\alpha, 1} g(x)
$$

when $0<r<1$. The term on the right side goes to zero as $r \longrightarrow 0$ if $\mathcal{M}_{\alpha, 1} g(x)<\infty$. This shows that $x$ is the Lebesgue point for $u$.

The set of the non-Lebesgue points is contained in

$$
E_{\infty}=\left\{x \in X: \mathcal{M}_{\alpha, 1} g(x)=\infty\right\}
$$

Note that

$$
\mathcal{M}_{\alpha, 1} g(x) \leq\left(\mathcal{M}_{\alpha p, 1} g^{p}(x)\right)^{1 / p}
$$

Let $1<q<p$. Choose $\alpha=q / p$. Inequality (4.5) and the weak type estimate (2.7) yield

$$
\begin{aligned}
\mathcal{H}_{\infty}^{n-q}\left(E_{\infty} \cap B(y, 1)\right) & \leq \mathcal{H}_{\infty}^{n-q}\left(\left\{x \in B(y, 1): \mathcal{M}_{q, 1} g^{p}(x)>\lambda^{p}\right\}\right) \\
& \leq c \lambda^{-p} \int_{X} g^{p} d \mu,
\end{aligned}
$$

for every $\lambda>0$. Letting $\lambda \longrightarrow \infty$ we see that

$$
\mathcal{H}_{\infty}^{n-q}\left(E_{\infty} \cap B(y, 1)\right)=0
$$

for every ball $B(y, 1)$ and hence $\mathcal{H}^{n-q}\left(E_{\infty}\right)=0$ for any $q<p$. This gives the desired estimate for the Haudorff dimension. The proof is complete. 


\section{Hölder quasicontinuity and approximation of Sobolev func- tions.}

In this section we assume that $u$ coincides with the representative $\widetilde{u}$ defined pointwise by (4.1). It follows from the proof of Lemma 3.6 that for every $0<\beta \leq 1$ the inequality

$$
|u(x)-u(y)| \leq c d(x, y)^{\beta}\left(u_{\beta, 4 d(x, y)}^{\#}(x)+u_{\beta, 4 d(x, y)}^{\#}(y)\right) .
$$

holds for every $x \neq y$. It may happen that the left hand side of (5.1) is of the indefinite form like $|\infty-\infty|$, then we adopt the convention $|\infty-\infty|=\infty$. In any case inequality (5.1) remains valid since $u(x)=$ $\pm \infty$ implies that $u_{\beta, R}^{\#}(x)=\infty$ for every $R>0$.

In particular, if $\left\|u_{\beta}^{\#}\right\|_{\infty}<\infty$, then (5.1) shows that $u$ is Hölder continuous. This is the content of Morrey's lemma, see [MS1, Theorem 4].

Denote

$$
E_{\lambda}=\left\{x \in X: u_{\beta}^{\#}(x)>\lambda\right\}, \quad \lambda>0 .
$$

Using (5.1) we see that $\left.u\right|_{X \backslash E_{\lambda}}$ is Hölder continuous with the exponent $\beta$. We can extend this function to a Hölder continuous function on $X$ using the McShane extension

$$
u(x)=\inf \left\{u(y)+2 \lambda d(x, y)^{\beta}: y \in X \backslash E_{\lambda}\right\},
$$

for every $x \in X$, see [Mc]. However, this does not guarantee that the extended function belongs to the Sobolev space $W^{1, p}(X)$ nor that it is close to the original function in the Sobolev norm. For that we need a Whitney type extension. In fact, we do not extend the function from the set $X \backslash E_{\lambda}$, but we smooth the function $u$ outside that set leaving the values of $u$ on $X \backslash E_{\lambda}$ unchanged. Thus our construction should be called the Whitney smoothing.

Now we are ready for the main result of the paper.

Theorem 5.3. Suppose that $u \in W^{1, p}(X)$ is defined pointwise by (4.1), $1<p \leq n$ and let $0<\beta \leq 1$. Then for every $\varepsilon>0$ there is a function $w$ and an open set $O$ such that

1) $u=w$ everywhere in $X \backslash O$,

2) $w \in W^{1, p}(X)$, and $w$ is Hölder continuous with the exponent $\beta$ on every bounded set in $X$, 
3) $\|u-w\|_{W^{1, p}(X)}<\varepsilon$,

4) $\mathcal{H}_{\infty}^{n-(1-\beta) p}(O)<\varepsilon$.

Proof. First suppose that the support of $u \in W^{1, p}(X)$ is contained in a ball

$$
\operatorname{supp} u \subset B\left(x_{0}, 1\right) \text {, }
$$

for some $x_{0} \in X$. The general case follows from a localization argument. Various constants $c$ that appear in the proof do not depend on $\lambda$.

Note that there is $\lambda_{0}>0$ such that for every $r>1$ and $x \in X$ we have

$$
r^{-\beta} f_{B(x, r)}\left|u-u_{B(x, r)}\right| d \mu<\lambda_{0}
$$

and hence $E_{\lambda} \subset B\left(x_{0}, 2\right)$ when $\lambda>\lambda_{0}$. Indeed, if the term on the left hand side of (5.4) is positive then $B(x, r) \cap B\left(x_{0}, 1\right) \neq \varnothing$. Thus by the doubling property we have

$$
\mu(B(x, r)) \geq c \mu\left(B\left(x_{0}, 1\right)\right)>0,
$$

for $r>1$ and estimate (5.4) follows easily.

It is easy to verify that the set $E_{\lambda}$ defined by (5.2) is open.

Let $g \in D(u) \cap L^{p}(X)$. If $x \in E_{\lambda}$ and $\lambda>\lambda_{0}$, by (5.4), (3.11) and the Hölder inequality we obtain

$$
u_{\beta}^{\#}(x)=u_{\beta, 1}^{\#}(x) \leq c\left(\mathcal{M}_{(1-\beta) p, 1} g^{p}(x)\right)^{1 / p} \leq c\left(\mathcal{M} g^{p}(x)\right)^{1 / p} .
$$

The weak type estimate (2.4) shows that

$$
\mu\left(E_{\lambda}\right) \leq \mu\left(\left\{x \in X: \mathcal{M} g^{p}(x)>c \lambda^{p}\right\}\right) \leq c \lambda^{-p} \int_{X} g^{p} d \mu<\infty
$$

for every $\lambda>\lambda_{0}$.

We recall the following Whitney type covering theorem [MS2, Lemma 2.9] and [CW].

Lemma 5.7. Let $O \subset X$ be an open set such that $O \neq X$ and $\mu(O)<$ $\infty$. For given $C \geq 1$, let $r(x)=\operatorname{dist}(x, X \backslash O) /(2 C)$. Then there is $N \geq 1$ and a sequence $\left\{x_{i}\right\}$ such that, denoting $r\left(x_{i}\right)=r_{i}$, the following properties are true: 
1) The balls $B\left(x_{i}, r_{i} / 4\right)$ are pairwise disjoint.

2) $\bigcup_{i \in I} B\left(x_{i}, r_{i}\right)=O$.

3) $B\left(x_{i}, C r_{i}\right) \subset O$ for every $i=1,2, \ldots$

4) For every $i, x \in B\left(x_{i}, C r_{i}\right)$ implies that

$$
C r_{i} \leq \operatorname{dist}(x, X \backslash O) \leq 3 C r_{i} .
$$

5) For every $i$, there is $y_{i} \in X \backslash O$ such that $d\left(x_{i}, y_{i}\right)<3 C r_{i}$.

6) $\sum_{i=1}^{\infty} \chi_{B\left(x_{i}, C r_{i}\right)} \leq N$.

The previous covering lemma enables us to construct a partition of unity, see [MS2, Lemma 2.16] or [Se, Lemma C.31]. Let $B\left(x_{i}, r_{i}\right)$, $i=1,2, \ldots$, be the Whitney covering of $E_{\lambda}$ constructed in Lemma 5.7 with $C=5$. Then there are non-negative functions $\left\{\varphi_{i}\right\}_{i=1}^{\infty}$ such that $\operatorname{supp} \varphi_{i} \subset B\left(x_{i}, 2 r_{i}\right), 0 \leq \varphi_{i}(x) \leq 1$ for every $x \in X$, every $\varphi_{i}$ is Lipschitz with the constant $c / r_{i}$ and

$$
\sum_{i=1}^{\infty} \varphi_{i}(x)=\chi_{E_{\lambda}}(x), \quad x \in X .
$$

We define the Whitney smoothing of $u$ by

$$
w(x)= \begin{cases}u(x), & x \in X \backslash E_{\lambda}, \\ \sum_{i=1}^{\infty} \varphi_{i}(x) u_{B\left(x_{i}, 2 r_{i}\right)}, & x \in E_{\lambda} .\end{cases}
$$

Note that since $E_{\lambda} \subset B\left(x_{0}, 2\right)$ for $\lambda>\lambda_{0}$, we have supp $w \subset B\left(x_{0}, 2\right)$. We prove the theorem with $O=E_{\lambda}$ for sufficiently large $\lambda$.

Claim 1) is a trivial consequence of the definition of $w$.

Claim 2). First we show that $w$ is Hölder continuous with the exponent $\beta$.

Suppose that $x \in E_{\lambda}$ and choose $\bar{x} \in X \backslash E_{\lambda}$ so that $d(x, \bar{x}) \leq$ $2 \operatorname{dist}\left(x, X \backslash E_{\lambda}\right)$. Then by (5.8) and (5.9) we have

$$
\begin{aligned}
|w(\bar{x})-w(x)| & =\left|\sum_{i=1}^{\infty} \varphi_{i}(x)\left(u(\bar{x})-u_{B\left(x_{i}, 2 r_{i}\right)}\right)\right| \\
& \leq \sum_{i \in I_{x}}\left|u(\bar{x})-u_{B\left(x_{i}, 2 r_{i}\right)}\right|
\end{aligned}
$$


where $i \in I_{x}$ if and only if $x \in \operatorname{supp} \varphi_{i}$. A straightforward calculation, using the properties of the partition of unity, shows that for every $i \in I_{x}$ we have $B\left(x_{i}, 2 r_{i}\right) \subset B\left(\bar{x}, 50 r_{i}\right)$. Hence the argument similar to that in the proof of (3.9) gives

$$
\left|u(\bar{x})-u_{B\left(x_{i}, 2 r_{i}\right)}\right| \leq c r_{i}^{\beta} u_{\beta}^{\#}(\bar{x}) .
$$

Since the overlap of the balls $B\left(x_{i}, 2 r_{i}\right)$ is uniformly bounded by Lemma 5.7.6), we see that the cardinality of $I_{x}$ is uniformly bounded. By Lemma 5.7.4) we see that $r_{i}, i \in I_{x}$, is comparable to $\operatorname{dist}(x, \bar{x})$. Using (5.10), (5.11) and recalling that $u_{\beta}^{\#}(\bar{x}) \leq \lambda$ for $\bar{x} \in X \backslash E_{\lambda}$, we arrive at

$$
|w(\bar{x})-w(x)| \leq c d(\bar{x}, x)^{\beta} u_{\beta}^{\#}(\bar{x}) \leq c \lambda d(\bar{x}, x)^{\beta} .
$$

We show that

$$
|w(x)-w(y)| \leq c \lambda d(x, y)^{\beta} .
$$

for all $x, y \in X$. We divide the proof into several cases.

First suppose that $x, y \in E_{\lambda}$ and let

$$
\gamma=\min \left\{\operatorname{dist}\left(x, X \backslash E_{\lambda}\right), \operatorname{dist}\left(y, X \backslash E_{\lambda}\right)\right\} .
$$

If $d(x, y) \geq \gamma$, then (5.12), the fact that $\bar{x}, \bar{y} \in X \backslash E_{\lambda}$ and (5.1) imply

$$
\begin{aligned}
|w(x)-w(y)| & \leq|w(x)-w(\bar{x})|+|u(\bar{x})-u(\bar{y})|+|w(\bar{y})-w(y)| \\
& \leq c \lambda\left(d(x, \bar{x})^{\beta}+d(\bar{x}, \bar{y})^{\beta}+d(\bar{y}, y)^{\beta}\right) \\
& \leq c \lambda d(x, y)^{\beta} .
\end{aligned}
$$

Suppose then that $x, y \in E_{\lambda}$ with $d(x, y) \leq \gamma$. By (5.8) we have

$$
\sum_{i=1}^{\infty}\left(\varphi_{i}(x)-\varphi_{i}(y)\right)=0 .
$$

Hence we obtain

$$
\begin{aligned}
|w(x)-w(y)| & =\left|\sum_{i=1}^{\infty}\left(\varphi_{i}(x) u_{B\left(x_{i}, 2 r_{i}\right)}-\varphi_{i}(y) u_{B\left(x_{i}, 2 r_{i}\right)}\right)\right| \\
& =\left|\sum_{i=1}^{\infty}\left(\varphi_{i}(x)-\varphi_{i}(y)\right)\left(u(\bar{x})-u_{B\left(x_{i}, 2 r_{i}\right)}\right)\right| \\
& \leq c d(x, y) \sum_{i \in I_{x} \cup I_{y}} r_{i}^{-1}\left|u(\bar{x})-u_{B\left(x_{i}, 2 r_{i}\right)}\right| .
\end{aligned}
$$


Using the same argument as in (5.11) we see that

$$
|w(x)-w(y)| \leq c d(x, y)^{\beta} \sum_{i \in I_{x} \cup I_{y}} \frac{d(x, y)^{1-\beta}}{r_{i}^{1-\beta}} u_{\beta}^{\#}(\bar{x}) \leq c \lambda d(x, y)^{\beta} .
$$

The last inequality follows from the fact that $r_{i}, i \in I_{x} \cup I_{y}$, is comparable to $\gamma$.

If $x, y \in X \backslash E_{\lambda}$, then the claim follows from (5.1). If $x \in E_{\lambda}$ and $y \in X \backslash E_{\lambda}$, then

$$
|w(x)-w(y)| \leq|w(x)-w(\bar{x})|+|u(\bar{x})-u(y)|
$$

and the claim follows from (5.12) and (5.1). This proves the Hölder continuity of $w$.

Then we prove that $w \in W^{1, p}(X)$. To this end, it suffices to show that $w \in L^{p}(X)$ and that for $g \in D(u) \cap L^{p}(X)$ we have $\mathcal{M} g \in$ $D(w) \cap L^{p}(X)$.

First we observe that

$$
\int_{E_{\lambda}}|w|^{p} d \mu \leq c \sum_{i=1}^{\infty} \int_{B\left(x_{i}, 2 r_{i}\right)}\left|u_{B\left(x_{i}, 2 r_{i}\right)}\right|^{p} d \mu \leq c \int_{E_{\lambda}}|u|^{p} d \mu .
$$

In both inequalities we applied the uniform bound for the overlapping number of the balls $B\left(x_{i}, 2 r_{i}\right) \subset E_{\lambda}$ (Lemma 5.7.6) and 5.7.3)). Since $w(x)=u(x)$ for every $x \in X \backslash E_{\lambda}$, we see that $w \in L^{p}(X)$.

Let $g \in D(u) \cap L^{p}(X)$. Then for almost every $x, y \in X \backslash E_{\lambda}$ we have

$$
|w(x)-w(y)|=|u(x)-u(y)| \leq d(x, y)(g(x)+g(y)) .
$$

For almost every $x, y \in E_{\lambda}$ with $d(x, y) \leq \gamma(c f$. (5.13)) the calculation as in (5.14) gives

$$
|w(x)-w(y)| \leq c d(x, y) \sum_{i \in I_{x} \cup I_{y}} r_{i}^{-1}\left|u(x)-u_{B\left(x_{i}, 2 r_{i}\right)}\right|
$$

Since $d(x, y)$ is small enough, we have $B\left(x_{i}, 2 r_{i}\right) \subset B\left(x, 100 r_{i}\right)$ whenever $i \in I_{x} \cup I_{y}$. Then by the Poincaré inequality and the doubling condition we obtain

$$
\left|u(x)-u_{B\left(x_{i}, 2 r_{i}\right)}\right| \leq c r_{i} \mathcal{M} g(x) .
$$


Since the cardinality of $I_{x} \cup I_{y}$ is bounded, by (5.16) we obtain

$$
|w(x)-w(y)| \leq c d(x, y) \mathcal{M} g(x) .
$$

For almost every $x, y \in E_{\lambda}$ with $d(x, y) \geq \gamma$, using the same argument as in (5.17), we have

$$
\begin{aligned}
|w(x)-w(y)|= & \mid \sum_{i=1}^{\infty}\left(\varphi_{i}(x)\left(u_{B\left(x_{i}, 2 r_{i}\right)}-u(x)\right)\right. \\
& \left.-\varphi_{i}(y)\left(u_{B\left(x_{i}, 2 r_{i}\right)}-u(y)\right)\right)+(u(x)-u(y)) \mid \\
\leq & \sum_{i \in I_{x}}\left|u(x)-u_{B\left(x_{i}, 2 r_{i}\right)}\right|+\sum_{i \in I_{y}}\left|u(y)-u_{B\left(x_{i}, 2 r_{i}\right)}\right| \\
& +|u(x)-u(y)| \\
\leq & c \operatorname{dist}\left(x, X \backslash E_{\lambda}\right) \mathcal{M} g(x)+c \operatorname{dist}\left(y, X \backslash E_{\lambda}\right) \mathcal{M} g(y) \\
& +d(x, y)(g(x)+g(y)) \\
\leq & c d(x, y)(\mathcal{M} g(x)+\mathcal{M} g(y)) .
\end{aligned}
$$

If either $x \in X \backslash E_{\lambda}$ or $y \in X \backslash E_{\lambda}$, then the proof is similar. We conclude that

$$
|w(x)-w(y)| \leq c d(x, y)(\mathcal{M} g(x)+\mathcal{M} g(y)),
$$

for almost every $x, y \in X$. By the Hardy-Littlewood theorem (Proposition 2.3) we obtain $\mathcal{M} g \in L^{p}(X)$. This shows that $u \in W^{1, p}(X)$.

Claim 3). Then we prove that $w \longrightarrow u$ in $W^{1, p}(X)$ as $\lambda \longrightarrow \infty$. Since $\mu\left(E_{\lambda}\right) \longrightarrow 0$ as $\lambda \longrightarrow \infty$, we conclude using inequalities (5.15) and (5.6) that $\|u-w\|_{L^{p}(X)} \longrightarrow 0$ as $\lambda \longrightarrow \infty$. Now we take care about the "gradient" estimates.

Let $g \in D(u) \cap L^{p}(X)$. Inequalities (5.18) and (5.17) imply that for a suitable constant $c$ the function $g_{\lambda}=c(\mathcal{M g}) \chi_{E_{\lambda}}$ satisfies $g_{\lambda} \in$ $D(u-w) \cap L^{p}(X)$. Since $\left\|g_{\lambda}\right\|_{L^{p}(X)} \longrightarrow 0$, as $\lambda \longrightarrow 0$ we conclude that $\|u-w\|_{L^{1, p}(X)} \longrightarrow 0$ as $\lambda \longrightarrow \infty$.

Claim 4). The claim follows from Lemma 2.6 and the fact that for $\lambda>\lambda_{0}$ we have

$$
E_{\lambda} \subset\left\{x \in B\left(x_{0}, 2\right): \mathcal{M}_{(1-\beta) p, 1} g^{p}(x)>c \lambda^{p}\right\},
$$


for some $c>0$, see (5.2) and (5.5). This completes the proof in the case when the support of $u$ lies in a ball.

The case of general $u \in W^{1, p}(X)$ will be deduced from the case when $u$ has the support in a ball via a partition of unity. First we need a lemma which shows that the multiplication by a bounded Lipschitz function is a bounded operator in the Sobolev norm. The following lemma is in some sense a generalization of the Leibniz differentiation rule.

Lemma 5.20. Let $u \in W^{1, p}(X)$ and $\varphi$ be a bounded Lipschitz function. Then $u \varphi \in W^{1, p}(X)$. Moreover, if $L$ is a Lipschitz constant of $\varphi$ and $\operatorname{supp} \varphi=K$, then

$$
\left(g\|\varphi\|_{\infty}+L|u|\right) \chi_{K} \in D(u \varphi) \cap L^{p}(X),
$$

for every $g \in D(u) \cap L^{p}(X)$.

Proof. The triangle inequality implies that

$$
|u(x) \varphi(x)-u(y) \varphi(y)| \leq d(x, y)((g(x)+g(y))|\varphi(x)|+L|u(y)|)
$$

and

$$
|u(x) \varphi(x)-u(y) \varphi(y)| \leq d(x, y)((g(x)+g(y))|\varphi(y)|+L|u(x)|) .
$$

Now it suffices to consider four easy cases depending on whether each of the points $x, y$ belongs to $K$ or not. This completes the proof.

Now we are ready to complete the proof of Theorem 5.3. Let $B\left(x_{i}, 1 / 4\right), i=1,2, \ldots$, be a maximal family of pairwise disjoint balls in $X$. Then by maximality $X \subset \bigcup_{i=1}^{\infty} B\left(x_{i}, 1 / 2\right)$. Let $\left\{\varphi_{i}\right\}_{i=1}^{\infty}$ be a partition of unity such that $\operatorname{supp} \varphi_{i} \subset B\left(x_{i}, 1\right), 0 \leq \varphi_{i}(x) \leq 1$ for every $x \in X$,

$$
\sum_{i=1}^{\infty} \varphi_{i}(x)=1,
$$

for every $x \in X$, and $\varphi_{i}, i=1,2, \ldots$, are Lipschitz continuous with the same Lipschitz constant.

Suppose that $u \in W^{1, p}(X)$. Then

$$
u(x)=\sum_{i=1}^{\infty} u \varphi_{i}(x),
$$


for every $x \in X$. Lemma 5.20 implies that the series converges also in the Sobolev norm. Let $\varepsilon>0$. Clearly supp $u \varphi_{i} \subset B\left(x_{i}, 1\right), i=1,2, \ldots$ Let $w_{i} \in W^{1, p}(X), i=1,2, \ldots$, be a Hölder continuous function with the exponent $\beta$ such that

$$
\begin{gathered}
\mathcal{H}_{\infty}^{n-(1-\beta) p}\left(\left\{x \in X: w_{i}(x) \neq u \varphi_{i}(x)\right\}\right) \leq 2^{-i} \varepsilon, \\
\left\|w_{i}-u \varphi_{i}\right\|_{W^{1, p}(X)} \leq 2^{-i} \varepsilon
\end{gathered}
$$

and

$$
\operatorname{supp} w_{i} \subset B\left(x_{i}, 2\right) .
$$

Then it is easy to see that

$$
w=\sum_{i=1}^{\infty} w_{i}
$$

has the desired properties. The proof of Theorem 5.3 is complete.

REMARKS 5.21. 1) The case $\beta=1$ of Theorem 5.3 has been previously proved in [H1]. This case is much easier, since it suffices to use the McShane extension [Mc]. Indeed, a locally Lipschitz function belongs to the Sobolev space $W^{1, p}(X)$ by the definition.

2) Using Lemma 3.15 and (5.19) we see that estimate (4) in Theorem 5.3 may be replaced by

$$
\mathrm{C}_{(1-\beta) p}(O)<\varepsilon,
$$

provided $\beta<1-1 / p$.

3) If the measure is Ahlfors-David regular, which means that there are $n>0$ and $c \geq 1$ so that

$$
c^{-1} r^{n} \leq \mu(B(x, r)) \leq c r^{n}, \quad x \in X, 0<r \leq \operatorname{diam}(X),
$$

then the function $w$ in Theorem 5.3 can be chosen to be globally Hölder continuous on $X$. Indeed, then the boundedness assumption in Lemma 2.6 is not needed. In addition, observe that we do not require that the space is bounded in (5.22). 


\section{References.}

[BZ] Bagby, T., Ziemer, W. P., Pointwise differentiablity and absolute continuity. Trans. Amer. Math. Soc. 191 (1974), 129-148.

[BH] Bojarski, B., Hajłasz, P., Pointwise inequalities for Sobolev functions and some applications. Studia Math. 106 (1993), 77-92.

[BHS] Bojarski, B., Hajłasz, P., Strzelecki, P., Pointwise inequalities for Sobolev functions revisited. Preprint, 1998.

[C] Calderón, A. P., Estimates for singular integral operators in terms of maximal functions. Studia Math. 44 (1972), 563-582.

[CZ] Calderón, A. P., Zygmund, A., Local properties of solutions to elliptic partial differential equations. Studia Math. 20 (1961), 171-225.

[CDG] Capogna, L., Danielli, D., Garofalo, N., Subelliptic mollifiers and a basic pointwise estimate of Poincaré type. Math. Z. 226 (1997), 147-154.

[Ch] Christ, M., Lectures on Singular Integral Operators. Amer. Math. Soc. Regional Conference Series in Math. 77, 1989.

[CW] Coifman, R. R., Weiss, G., Analyse Harmonique Non-Commutative sur Certain Espaces Homogenés. Lecture Notes in Math. 242, SpringerVerlag, 1971.

[DS] DeVore, R., Sharpley, R. C., Maximal functions measuring smoothness. Mem. Amer. Math. Soc. Vol. 47. Number 293, 1984.

[EG] Evans, L. C., Gariepy, R. F., Measure Theory and Fine Properties of Functions. CRC Press, 1992.

[FZ] Federer, H., Ziemer, W., The Lebesgue set of a function whose distribution derivatives are $p$-th power summable. Indiana Univ. Math. J. 22 (1972), 139-158.

[FHK] Franchi, B., Hajłasz, P., Koskela, P., Definitions of the Sobolev classes on metric spaces. Preprint, 1998.

[FLW1] Franchi, B., Lu, G., Wheeden, R., A relationship between Poincaré type inequalities and representation formulas in spaces of homogeneous type. Int. Mat. Res. Notices (1996), 1-14.

[FLW2] Franchi, B., Lu, G., Wheeden, R., Representation formulas and weighted Poincaré inequalities for Hörmander vector fields. Ann. Inst. Fourier 45 (1995), 577-604.

[FSS] Franchi, B., Serapioni, R., Serra Cassano, F., Approximation and imbedding theorems for weighted Sobolev spaces associated with Lipschitz continuous vector fields. Bollettino U.M.I. 11-B (1997), 83-117.

[GN1] Garofalo, N., Nhieu, D. M., Isoperimetirc and Sobolev inequalities for Carnot-Carathéodory spaces and the existence of minimal surfaces. Comm. Pure Appl. Math. 49 (1996), 1081-1144. 
[GN2] Garofalo, N., Nhieu, D. M., Lipschitz continuity, global smooth approximations and extensions theorems for Sobolev functions in CarnotCarathéodory spaces. Preprint, 1996.

[H1] Hajłasz, P., Sobolev spaces on an arbitrary metric space. Potential Anal. 6 (1996), 403-415.

[H2] Hajłasz, P., Geometric approach to Sobolev spaces and badly degenerate elliptic equations. The Proceedings of Banach Center Minisemester: Nonlinear Analysis and Applications. GAKUTO International Series; Mathematical Sciences and Applications. 7 (1995), 141-168.

[HK1] Hajłasz, P., Koskela, P., Sobolev meets Poincaré. C. R. Acad. Sci. Paris 320 (1995), 1211-1215.

[HK2] Hajłasz, P., Koskela, P., Sobolev met Poincaré. Preprint, 1998.

[HM] Hajłasz, P., Martio, O., Traces of Sobolev functions on fractal type sets and characterization of extension domains. J. Funct. Anal. 143 (1997), 221-246.

[HeK] Heinonen, J., Koskela, J., Quasiconformal maps on metric spaces with controlled geometry. To appear in Acta Math.

$[\mathrm{J}]$ Jerison, D., The Poincaré inequality for vector fields satisfying Hörmander's condition. Duke Math. J. 53 (1986), 503-523.

[K] Kałamajska, A., On compactness of embedding for Sobolev spaces defined on metric spaces. To appear in Ann. Acad. Sci. Fenn. Math.

[KM] Kinnunen, J., Martio, O., The Sobolev capacity on metric spaces. Ann. Acad. Sci. Fenn. Math. 21 (1996), 367-382.

[KMc] Koskela, P., MacManus, P., Sobolev classes and quasisymmetric maps. To appear in Studia Math.

[Li] Liu, F.-C., A Lusin type property of Sobolev functions. Indiana Univ. Math. J. 26 (1977), 645-651.

[MS1] Macías, R. A., Segovia, C., Lipschitz functions on spaces of homogeneous type. Advances in Math. 33 (1979), 257-270.

[MS2] Macías, R. A., Segovia, C., A decomposition into atoms of distributions on spaces of homogeneous type. Advances in Math. 33 (1979), 271-309.

[Ma1] Malý, J., Hölder type quasicontinuity. Potential Anal. 2 (1993), 249254.

[Ma2] Malý, J., The area formula for $W^{1, n}$-mappings. Comment. Math. Univ. Carolinae 35 (1994), 291-298.

[MM] Malý, J., Martio, O., Lusin's condition (N) and mappings of the class $W^{1, n}$ J. Reine Angew. Math. 458 (1995), 19-36.

[Mc] McShane, E. J., Extension of range of functions. Bull. Amer. Math. Soc. 40 (1934), 837-842.

[MZ] Michael, J. L., Ziemer, W. P., A Lusin type approximation of Sobolev 
functions by smooth functions. Contemporary Mathematics, Amer. Math. Soc. 42 (1985), 135-167.

[Se] Semmes, S., Finding curves on general spaces through quantitative topology with applications to Sobolev and Poincaré inequalities. Selecta Math. (N.S.) 2 (1996), 155-295.

[St] Stein, E. M., Harmonic Analysis: Real Variable Methods, Orthogonality and Oscillatory Integrals. Princeton Univ. Press, 1993.

[Vo] Vodop'yanov, S., Monotone functions and quasiconformal mappings on Carnot groups. Siberian Math. J. 37 (1996), 1113-1136.

[Z] Ziemer, W. P., Weakly Differentiable Functions. Springer-Verlag, 1989.

Recibido: 26 de junio de 1.997

Piotr Hajłasz*

Institute of Mathematics

Warsaw University

ul. Banacha 2

02-097 Warszawa, POLAND

hajlasz@mimuw.edu.pl

and

Juha Kinnunen $^{\dagger}$

Department of Mathematics

P.O. Box 4

FIN-00014 University of Helsinki, FINLAND

Juha.Kinnunen@Helsinki.Fi

\footnotetext{
* Supported by the KBN, grant 2-PO3A-034-08
}

$\dagger$ Supported by the Academy of Finland 